\title{
Liste des auditions du groupe PSN
}

(Les comptes rendus de ces auditions sont disponibles)

25 septembre 2008, Monsieur F. Le Carvennec, expert indépendant 2 octobre 2008, Messieurs D. Uhde et H. Richter (Thomson Allemagne)

16 octobre 2008, Messieurs J. M. Rodes et F. Berahou, INA 24 octobre 2008, Monsieur Jacques Perdereau, LNE

5 novembre 2008, Messieurs J.-M. Fontaine (LAM, Institut Jean Le Rond d'Alembert) et J. J. Wanègue, expert indépendant 14 novembre : Monsieur P. André, Plasmon France (Caen)

17 novembre 2008: Messieurs J. Raby et A. Fargeix, LETI (Grenoble)

17 décembre 2008 : Monsieur L. de Poix, entreprise MPO

19 décembre 2008: Messieurs A. Beaufort, T. Ledoux et L. Duplouy (BNF)

21 janvier 2009 : Madame F. Banat-Berger et Monsieur M.Jacobson (Direction des Archives de France) 
28 janvier 2009 : Madame M. Campana et Monsieur J. M. Besse (ministère de l'Industrie)

9 mars 2009 : Monsieur S. Deleonibus, CEA-LETI (Grenoble)

9 mars 2009 : Monsieur T. Ihashi (Bifrostec, Japon)

9 mars 2009 : Messieurs J. P. Gleyzes, M. Buégué, et Madame D. Boucon (CNES)

11 mars 2009 : Madame G. Pinson, Messieurs J. B. Henniart et C. Girard, Secrétariat d'État au Développement de la Région Capitale

2 avril 2009 : Monsieur L. Ranno, Département nanosciences, Institut Néel, UJF, Grenoble

20 mai 2009: Messieurs F. Daumas, directeur du CINES (Montpellier) ; M. Auffret et O. Rouchon, département archivage et diffusion du CINES

25 juin 2009 : Monsieur Christian Amatore, membre de l'Académie des sciences

25 juin 2009 : Monsieur Gilles Lachaud, directeur de l'Institut de Mathématiques de Luminy

29 juin 2009: Messieurs T. Ihashi (Bifrostec) et A. Inoué (Mitsubishi Chemicals)

22 octobre 2009 : Madame E. Dion et Monsieur D. Reizine (APHP, radiologie) 